Marquette University

e-Publications@Marquette

Biological Sciences Faculty Research and

Publications

Biological Sciences, Department of

$9-1994$

\title{
A Small Family of Elements with Long Inverted Repeats is Located Near Sites of Developmentally Regulated DNA Rearrangement in Tetrahymena thermophila
}

John M. Wells

Brandeis University

Jay Lee Edward Ellingson

Marquette University

Diana M. Catt

Marquette University

Patricia J. Berger

Marquette University

Kathleen M. Karrer

Marquette University, kathleen.karrer@marquette.edu

Follow this and additional works at: https://epublications.marquette.edu/bio_fac

Part of the Biology Commons

\section{Recommended Citation}

Wells, John M.; Ellingson, Jay Lee Edward; Catt, Diana M.; Berger, Patricia J.; and Karrer, Kathleen M., "A Small Family of Elements with Long Inverted Repeats is Located Near Sites of Developmentally Regulated DNA Rearrangement in Tetrahymena thermophila" (1994). Biological Sciences Faculty Research and Publications. 225.

https://epublications.marquette.edu/bio_fac/225 


\title{
A Small Family of Elements with Long Inverted Repeats Is Located near Sites of Developmentally Regulated DNA Rearrangement in Tetrahymena thermophila
}

\author{
JOHN M. WELLS, ${ }^{1} \dagger$ JAY L. E. ELLINGSON,${ }^{2}$ DIANA M. CATT, ${ }^{2} \ddagger$ PATRICIA J. BERGER, ${ }^{2}$ \\ AND KATHLEEN M. KARRER ${ }^{2 *}$ \\ Department of Biology, Brandeis University, Waltham, Massachusetts 02154, ${ }^{1}$ and \\ Department of Biology, Marquette University, Milwaukee, Wisconsin $53233^{2}$
}

Received 14 December 1993/Returned for modification 9 February 1994/Accepted 23 May 1994

\begin{abstract}
Extensive DNA rearrangement occurs during the development of the somatic macronucleus from the germ line micronucleus in ciliated protozoans. The micronuclear junctions and the macronuclear product of a developmentally regulated DNA rearrangement in Tetrahymena thermophila, TIr1, have been cloned. The intrachromosomal rearrangement joins sequences that are separated by more than $13 \mathbf{~ k b}$ in the micronucleus with the elimination of moderately repeated micronucleus-specific DNA sequences. There is a long, 825-bp, inverted repeat near the micronuclear junctions. The inverted repeat contains two different 19-bp tandem repeats. The 19-bp repeats are associated with each other and with DNA rearrangements at seven locations in the micronuclear genome. Southern blot analysis is consistent with the occurrence of the 19-bp repeats within pairs of larger repeated sequences. Another family member was isolated. The 19-mers in that clone are also in close proximity to a rearrangement junction. We propose that the 19-mers define a small family of developmentally regulated DNA rearrangements having elements with long inverted repeats near the junction sites. We discuss the possibility that transposable elements evolve by capture of molecular machinery required for essential cellular functions.
\end{abstract}

Ciliated protozoa have two different kinds of nuclei: diploid, germ line micronuclei and transcriptionally active macronuclei. During sexual reproduction (conjugation), the old macronucleus is degraded and a new macronucleus develops from a mitotic product of the zygotic micronucleus.

The developing macronucleus undergoes radical changes in genome organization. In the holotrichous ciliate Tetrahymena thermophila, there is a period of DNA replication which brings the DNA content of the macronucleus to 45 times the haploid DNA content of the micronucleus. When the developing nucleus is between $4 \mathrm{C}$ and $8 \mathrm{C}$, about $15 \%$ of the germ line DNA sequences are eliminated from the somatic macronucleus $(11,36,58)$. Since DNA sequence elimination is site specific, highly regular, and developmentally controlled, it is expected that DNA rearrangement involves the interaction of specific cis-acting sequences with the rearrangement machinery.

Developmentally regulated DNA rearrangements in $T$. thermophila fall into two classes with respect to the fate of the macronucleus-destined sequences which flank the eliminated DNA (reviewed in reference 56). The first class of rearrangements results in chromosome breakage. The five metacentric chromosomes in the zygotic micronucleus are fragmented in the developing macronucleus to produce 50 to 250 subchromosomal molecules. A well-characterized example of this type of rearrangement is one in which the single rRNA gene in the

${ }^{*}$ Corresponding author. Phone: (414) 288-1474. Fax: (414) 2887357. Electronic mail address (Internet): 6193KARRERK@vms.csd. mu.edu.

$\dagger$ Present address: Department of Biochemistry, Boston University School of Medicine, Edith Nourse Rogers Memorial Veterans Hospital, Bedford, MA 01730.

$\ddagger$ Present address: Department of Microbiology and Immunology, Indiana University School of Medicine, Indianapolis, IN 46202. micronucleus is excised from the chromosome and short stretches of DNA on either side of the gene are eliminated (55). Both the extrachromosomal rRNA genes (rDNA) and the new subchromosome-size molecules flanking the eliminated DNA acquire telomeres, which stabilize the ends of the molecules. A 15-bp chromosome breakage sequence (Cbs) is thought to be the cis-acting sequence for chromosome fragmentation. The Cbs, originally found at the fragmentation sites for the rDNA, occurs at numerous additional sites in the micronuclear genome where it is associated exclusively with chromosomal fragmentation sites (60). Cbs has been shown to be both necessary and sufficient for chromosome breakage of constructs microinjected into developing macronuclei (59).

The second class of rearrangements in $T$. thermophila are breakage and joining events in which DNA sequence elimination is accompanied by ligation of flanking sequences. Over 5,000 of these events occur per haploid genome $(2,29)$. The length of eliminated DNA sequences varies over an order of magnitude, ranging from $600 \mathrm{bp}$ to more than $10 \mathrm{~kb}(12,57)$. Four short deletions, M, R, a deletion near the calmodulin gene, and mse2.9, have been described in detail at the sequence level $(3,4,26,37)$. For the $M$ rearrangement, $A_{5} G_{5}$ polypurine tracts have been shown to be necessary and sufficient for DNA rearrangement (23). The polypurine tracts are outside the deleted DNA and direct DNA rearrangement at a distance of 41 to $54 \mathrm{bp}$. Short direct repeats at the in vivo junctions of $\mathbf{M}$ are not required for rearrangement but may play a role in fine tuning the choice of junction site (24). The $R$ rearrangement, the deletion near the calmodulin gene, and mse2.9 all lack polypurine tracts. cis-acting sequences for the latter three rearrangements have not yet been identified. Although the four deletion-ligation rearrangements studied thus far have different sequences at the rearrangement junctions, it is not expected that each of the 5,000 rearrangements in the $T$. thermophila genome has unique cis-acting sequences. 
It has been suggested that the rearrangements belong to several classes, each specified by different flanking sequences (5).

We describe here a breakage and joining rearrangement which also lacks $A_{5} G_{5}$ tracts and is structurally distinct from those described previously in that the rearrangement junctions are widely separated in the micronuclear genome. The rearrangement is named Tlr1, for Tetrahymena long repeat, with reference to an 825-bp inverted repeat sequence located near the junctions of the rearrangement. Within the long inverted repeat of Tlr1 are two different 19-bp tandem repeats. The 19-mers are associated with each other and with DNA rearrangements at seven sites in the genome. Southern blot analysis suggests that the 19-mers occur within pairs of longer repeated sequences. Another member of the family on which the micronucleus-specific 19-mer repeats are linked to macronucleus-retained DNA, as expected for a clone containing a rearrangement junction, has been cloned. The data support a model for a small family of three or four DNA rearrangements having long repeated sequences near the rearrangement junctions.

\section{MATERIALS AND METHODS}

Strains. The wild-type inbred strain BVII and the heterokaryon strains CU428, Mpr/Mpr (6mp-s, VII), CU441, Chx/ Chx (cy-s, VI), and CU354 $\mathrm{ChxA}_{2} / \mathrm{ChxA}_{2}$ (cy-s, IV) were obtained from P. Bruns. Strain C2-468-3 (III) was provided by E. Orias. Caryonidal lines MU4A-D and MU5A-D were isolated from a cross between CU428 and CU441. Lines A and $\mathrm{B}$ are the caryonidal lines derived from one exconjugant and lines $C$ and $D$ are from the other exconjugant.

Cloning. Macronuclear DNA and micronuclear DNA were isolated by a modification of the method of Gorovsky et al. (25) as described Capowski et al. (16). DNA was digested with appropriate restriction enzymes and size fractionated on 0.7 to $1.0 \%$ agarose gels. The level of the running buffer was lowered to the top of the gel. A trough was cut in the agarose below the DNA of the desired size and filled with $25 \%$ glycerol in running buffer. The DNA was run into the trough at $200 \mathrm{~V}$ for $2.5 \mathrm{~min}$ and then collected and stored. The trough was refilled with glycerol in running buffer, and the procedure was repeated. Several fractions were collected. The DNA was concentrated with an Elutip-D column (Schleicher \& Schuell) as per the manufacturer's instructions. DNA slot blot analysis was done to determine which fraction(s) contained the desired DNA fragment.

The left junction of the rearrangement was cloned as a 1,930-bp HindIII fragment of micronuclear DNA, IIC7 (36). In order to clone the macronuclear junction site, size-fractionated 4.4-kb $B g l \mathrm{II}-X b a \mathrm{I}$-digested macronuclear DNA was ligated to Bam HI-XbaI-digested pUC18. Approximately 500 colonies were screened by an in situ lysis method (47). The probe was the 0.9-kb HindIII-Sau3A fragment, IIC7.1a, which contains single-copy DNA from the micronuclear clone (48).

Micronuclear DNA from the right-end rearrangement site was isolated from a library of $4.0-\mathrm{kb} B g l \mathrm{II}$ fragments ligated to BamHI-digested pUC13. Approximately 500 colonies were screened with a 1.8-kb Sau3A fragment, Tlr1.mS-S. Tlr1.mS-S contains single-copy DNA from the right side of the rearrangement.

Micronuclear DNA clone Tlr2.rX-C was cloned from sizefractionated HindIII-EcoRI-digested micronuclear DNA ligated to BluescriptSK vector and transformed into Escherichia coli CES201 (54). Approximately 500 colonies were screened with the 525-bp ClaI-HindIII fragment from Tlr1.rB-H, which contains the 19-mer repeats, as a probe. The clone was propagated in XL1-Blue cells (Stratagene) for sequencing.

PCR amplification of inverted repeat sequences near the right junction. For PCR amplification of sequences near the right junction of Tlr1, micronuclear DNA was digested with $H$ indIII and EcoRI. Restriction fragments were circularized by ligation of the DNA in the presence of two complementary oligonucleotides, 5'-AGCTTGAGCTCTCGAGTCGAC GATCG-3' and 5'-AATTCGATCGTCGACTCGAGAGCT CA-3', which hybridize to each other to form a linker with $H$ indIII and EcoRI sticky ends. The DNA sequences between the BglII site of Tlr1.rB-H and the internal EcoRI site were amplified by PCR with oligonucleotides 1 and 7 (see Fig. 2) as the primers. Oligonucleotide 7 is present in the inverted repeat of Tlr1 and possibly at other sites in the genome. Oligonucleotide 1 , which hybridized to unique DNA, conferred specificity on the PCR.

Southern hybridization. Purified micronuclear DNA isolated from strain CU441 was digested with the appropriate restriction endonucleases, fractionated by electrophoresis through $0.8 \%$ agarose gels, and blotted by the alternate method in the GeneScreen Plus protocol manual. Probes were radioactively labelled by the random primer method (47). The nylon filters were washed in $0.1 \times \operatorname{SSC}(1 \times \operatorname{SSC}$ is $0.15 \mathrm{M} \mathrm{NaCl}$ plus $0.015 \mathrm{M}$ sodium citrate) and $0.1 \%$ sodium dodecyl sulfate (SDS) at $65^{\circ} \mathrm{C}$.

In-gel hybridization. Macronuclear DNA and micronuclear DNA fragments were fractionated by electrophoresis through $1.0 \%$ agarose gels. The gels were dried in a Bio-Rad slab dryer (model 483) for $2 \mathrm{~h}$ at $60^{\circ} \mathrm{C}$. Dried-gel hybridizations of $19 \mathrm{~A}$ and 19B to digested genomic DNA were performed (20). The probes were end labelled with $\mathrm{T} 4$ polynucleotide kinase and put over a Sephadex G-50 spin column to separate the probe from the unincorporated nucleotides (47). 19A and 19B probes were hybridized in a $6 \times$ SSPE $(1 \times$ SSPE is $0.18 \mathrm{M} \mathrm{NaCl}, 10$ $\mathrm{mM} \mathrm{NaPO}$, and $1 \mathrm{mM}$ EDTA [pH 7.7]) solution at 37 and $42^{\circ} \mathrm{C}$, respectively, in a shaking water bath for $18 \mathrm{~h}$. The gels were washed with tetramethylammonium chloride solution [3 $\mathrm{M}\left(\mathrm{CH}_{3}\right)_{4} \mathrm{NCl}, 50 \mathrm{mM}$ Tris-Cl (pH 8.0), 2 mM EDTA, $1 \mathrm{mg}$ of SDS per $\mathrm{ml}$ ], which minimizes the effect of GC content on the dissociation temperature $\left(T_{d}\right)(53)$. Washes in tetramethylammonium chloride were done at $55^{\circ} \mathrm{C}\left(T_{d}-3^{\circ} \mathrm{C}\right)$, which allows hybridization of the oligonucleotide to only perfectly matched genomic sequences.

DNA sequencing. Double-stranded DNA sequencing was done by the Sanger dideoxynucleotide sequencing method, as previously described (1). Single-stranded DNA for sequencing was produced from Bluescript plasmids in XL1-Blue cells by using helper phage M13K07 (Stratagene) per the manufacturer's instructions. Direct sequencing of the right junction PCR product from two independent PCR amplifications was done as described by Klobutcher et al. (39). All micronuclear DNA was sequenced in both directions, and macronuclear DNA was sequenced at least twice.

Nucleotide sequence accession numbers. The left and right junction sequences of the Tlr1 rearrangement have been deposited in the GenBank database (accession numbers L25253 and L25254, respectively).

\section{RESULTS}

A developmentally regulated DNA rearrangement in $T$. thermophila, TIr1, results in elimination of DNA from the macronuclear genome with ligation of the flanking sequences. The 1.9-kb clone pTtIIC7 (Fig. 1) was isolated from a library of T. thermophila micronuclear DNA. The subclone pTtIIC7.2 


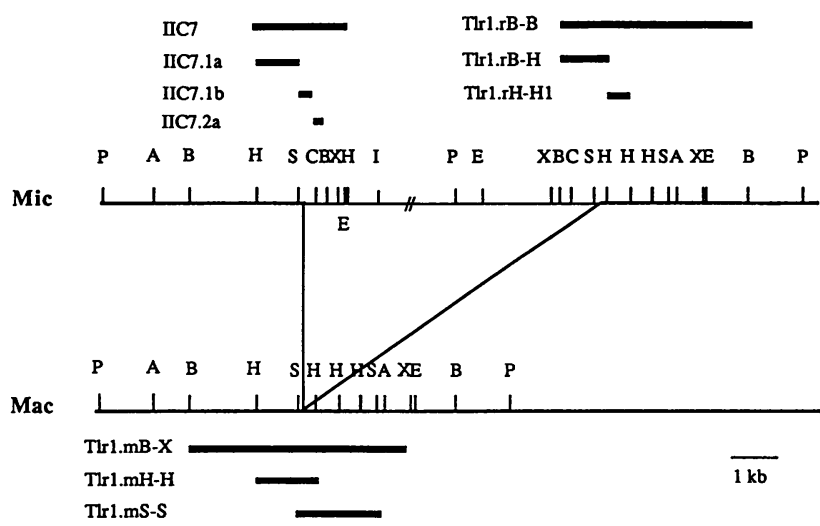

FIG. 1. Restriction maps of the micronuclear (Mic) and macronuclear (Mac) genomes in the vicinity of the Tlr1 rearrangement. Cloned sequences are indicated by the bars. Restriction sites: P, HpaI; A, HaeIII; B, BglII; H, HindIII; S, Sau3A; C, ClaI; X, XbaI; I, HindII; E, EcoRI. Additional Sau3A sites which are not shown on the map are present. // indicates that there is more than $13 \mathrm{~kb}$ of micronuclear DNA between the rearrangement junctions which has not been mapped in detail.

belonged to a family of repeated micronucleus-specific sequences $(36,48)$. The subclone pTtIIC7.1a contained singlecopy DNA which hybridized to a 1.9-kb fragment in Southern blots of HindIII-digested micronuclear DNA and to a $1.1-\mathrm{kb}$ fragment in macronuclear DNA. The simplest explanation for the difference in size between the micronuclear and macronuclear HindIII fragments was that the micronuclear HindIII fragment undergoes rearrangement during development of the macronucleus. IIC7 was arbitrarily designated as the left end of the Tlr1 rearrangement.

In order to determine the DNA sequence at the junction sites, it was necessary to obtain the micronuclear DNA representing the left and right ends of the rearrangement site and the macronuclear DNA which is the product of the rearrangement. The left and right junctions are a large, but undetermined, distance apart (data presented below), and the presence of repeated sequences in the eliminated DNA precluded a chromosome walk from the left to the right micronuclear junction. Therefore, a chromosome jump across the deleted DNA was done by isolating the macronuclear junction fragment and using a subclone of it to identify the micronuclear right junction clone.

Genomic restriction maps of the micronuclear DNA in the region of the left end of the rearrangement and of the macronuclear DNA were constructed by using IIC7.1a as a probe (Fig. 1). The mapping data suggested that the rearrangement junction was present on a 4.4-kb BglII-XbaI fragment in macronuclear DNA. The macronuclear fragment was isolated from $T$. thermophila BVII by screening a minilibrary of sizefractioned macronuclear DNA digested with $B g l \mathrm{II}$ and $X b a \mathrm{I}$, with IIC7.1a as the probe. This clone, designated Tlr1.mB-X, was digested with restriction enzymes and shown to contain the HindIII, Sau3A, and HaeIII sites predicted from the genomic map of macronuclear DNA. The 1.1-kb HindIII fragment containing the rearrangement junction, $\mathrm{Tl} 1 . \mathrm{mH}-\mathrm{H}$, was subcloned. It hybridized to the expected 1.9- and 1.1-kb HindIII fragments on Southern blots of micronuclear and macronuclear DNA, respectively.

The strategy used in cloning the right side of the IIC7 rearrangement was similar to the one used to clone the macronuclear DNA. A 4.0-kb BglII restriction fragment in micronuclear DNA was identified by hybridization with the 1.8-kb Sau3A subclone, Tlr1.mS-S. The cloned fragment, designated Tlr1.rB-B, had seven restriction sites identical to those in Tlr1.mB-X and $\sim 800$ bp of DNA not present in the macronuclear clone. The right junction of the rearrangement was located in the 844-bp BglII-HindIII fragment, Tlr1.rB-H.

The DNA sequences of the left junction clone IIC7 and the right junction clones Tlr1.rB-H and Tlr1.rH-H1 were determined (Fig. 2). Comparison with the sequence of the macronuclear clone, Tlr1.mH-H, confirmed the ligation event indicated by the restriction maps and unambiguously identified the junction site in strain BVII.

The TIr1 rearrangement is intrachromosomal. The junctions of Tlr1 are more widely separated in the micronuclear genome than those of breakage and joining rearrangements in T. thermophila which have been studied previously. Genomic restriction maps of micronuclear DNA were constructed by using pTtIIC7.1a and Tlr1.rH-H1 as hybridization probes (Fig. 1). In no case did the two probes hybridize to a restriction fragment of the same size. Hybridization of micronuclear DNA with oligonucleotide 1 (Fig. 2B) showed that the right junction is on a $12-\mathrm{kb}$ HindIII fragment, with only $51 \mathrm{bp}$ of DNA between the right junction and the HindIII site of Tlr1.rB-H. Since there is an additional $891 \mathrm{bp}$ of micronucleus-specific DNA between the left junction of the rearrangement and the HindIII site at the end of the IIC7 fragment, the left and right junctions of the Tlr1 rearrangement are separated by at least $13 \mathrm{~kb}$ in the micronucleus.

The relatively long distance between the right and left junctions of Tlr1 raised the question of whether the Tlr1 rearrangement is intrachromosomal. Linkage of the left and the right junctions of the Tlr1 rearrangement was demonstrated by PCR analysis of nullisomic strains of $T$. thermophila. Nullisomic strains are heterokaryons which have an intact macronuclear genome but lack one or more pairs of chromosomes in the micronucleus (14). The left junction of Tlr1 was PCR amplified with oligonucleotides 2 and 3 (Fig. 2A) as primers. Oligonucleotide 2 is located within single-copy DNA and conferred site specificity on the reaction. Oligonucleotide 3 , located within micronucleus-specific DNA, was chosen to obtain the PCR product which resulted from amplification of micronuclear, and not macronuclear, DNA sequences. Wholecell DNAs from diploid cells and from all nullisomic cell lines except those which lack chromosome 3 have the expected 1,726-bp PCR product (18). The 786-bp right junction fragment generated in a PCR with oligonucleotides 4 and 5 (Fig. 2B) was similarly mapped to chromosome 3 . Thus, the Tlr1 rearrangement is intrachromosomal.

Variability in the junction sites of the Tlr1 rearrangement. Southern blot analysis of DNA from caryonidal strains was done in order to assess the degree of variability of the Tlr1 rearrangement. The four caryonidal strains isolated from a mating pair of $T$. thermophila are genetically identical but developmentally independent (13). DNA rearrangement occurs after the genome has undergone one or two rounds of DNA replication $(2,11)$, when there are four to eight copies of the genome present in the macronuclear anlagen. Thus, for rearrangements having alternate junctions, a single macronucleus may produce more than one rearrangement product (5).

Two sets of caryonidal strains were isolated from a mating between strains CU428 and CU441. The cell lines were grown only to mid-log phase before preparation of DNA in order to minimize selection of particular variants due to possible differences in growth rate among the vegetative progeny. Thus, the DNA from the caryonidal lines was expected to contain all of the rearrangement products that were produced in the 


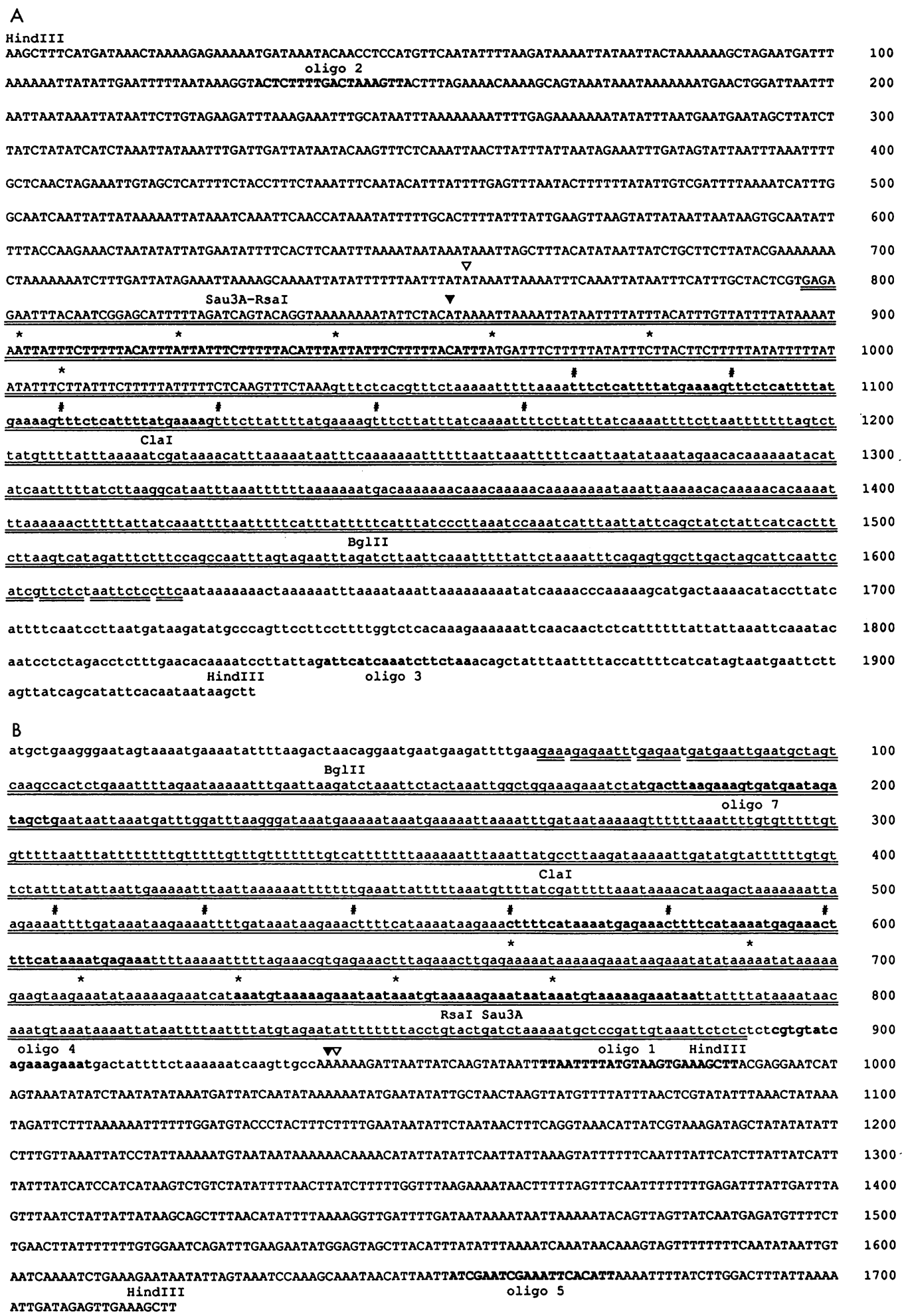

FIG. 2. DNA sequence of the left junction (A) and the right junction (B) of the Tlr1 rearrangement. The left junction is the sequence of pTtIIC7. The right junction is sequence of PCR product, pTtTlr1.rB-H, and pTtTlr1.rH-H1. Uppercase letters designate sequence in both micronuclear and macronuclear DNA, and lowercase letters represent DNA which is deleted from the macronucleus in strain BVII. Alternate junction sites in strains CU354 (filled arrowhead) and C2-468-3 (open arrowhead) result in the deletion of more DNA than in the major rearrangement. Alternate junction sites occur either 5' or 3' to the indicated adenines, an ambiguity due to the location of the right junction within a run of adenines. Double underlining indicates the 825-bp inverted repeat. Symbols: initial nucleotides of the 19A $(*)$ and 19B (\#) tandem repeats. Boldface indicates the oligonucleotides referred to in the text, including the perfect 19A and 19B repeats. Oligonucleotides of the sequence as written are numbered above the sequence, and oligonucleotides of the complementary sequence are numbered below the sequence. 

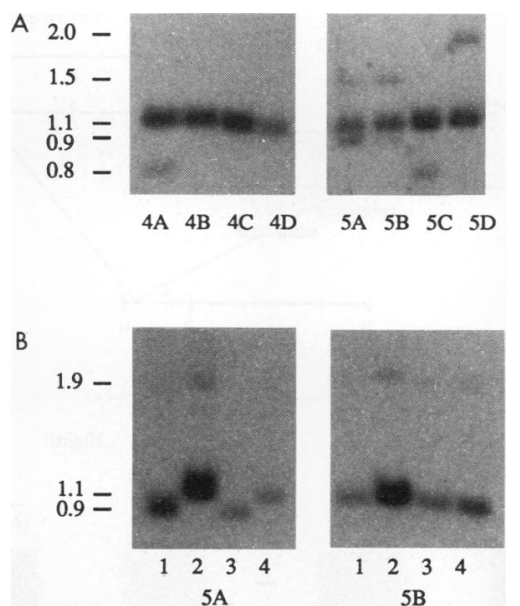

FIG. 3. Southern blots of macronuclear DNA isolated from two sets of caryonidal T. thermophila strains (A) and whole-cell DNA from subclones of the caryonidal strains 5A and 5B (B) following phenotypic assortment. DNA was digested with HindIII, and Southern blots were probed with IIC7.1a. The faint bands at $1.9 \mathrm{~kb}$ in panel $\mathrm{B}$ are the result of hybridization of the probe to micronuclear DNA. Numbers at the left indicate the approximate sizes (in kilobases) of the fragments.

developing macronuclear anlagen of the progenitor cell. Figure $3 \mathrm{~A}$ shows that all eight cell lines from two caryonidal sets tested contained the 1.1-kb macronuclear HindIII fragment as the major product of the rearrangement. Five of the eight cell lines also contained alternative rearrangement products represented by $0.8-, 0.9-, 1.5-$, and $2.0-\mathrm{kb}$ HindIII fragments.

The 0.8-, 0.9-, and $1.5-\mathrm{kb}$ products were bona fide alternative rearrangements by the criterion that they undergo phenotypic assortment in vegetatively dividing cells. The macronucleus of $T$. thermophila has no functional centromeres. Immediately following conjugation of cells with two different alleles, the macronucleus is heterozygous and contains multiple copies of both alleles. Through subsequent amitotic macronuclear divisions, the macronuclear chromosomes are apparently partitioned randomly and thus unequally to the progeny cells. Clonal cell lines established after 100 to 200 vegetative fissions are pure for one allele or the other. This phenomenon, called phenotypic assortment (13), has been established genetically for over 90 loci. The cell lines MU5A and MU5B were grown for 100 fissions. Clonal cell lines were established from the vegetative progeny. In the case of the progeny of strain MU5A, two of the subclones had assorted for the rearrangement product which has a 1.1-kb macronuclear HindIII fragment, and two had assorted for the 0.9-kb product. In other experiments, a cell line which has only the 1.5 -kb fragment has been isolated. Assortment for the 2.0-kb HindIII fragment has not yet been observed.

A 0.8- and a 0.9-kb HindIII fragment encompassing the Tlr1 macronuclear junction have been cloned from strains C2-468-3 and CU354, respectively. Sequence analysis confirmed that they represent alternative versions of the Tlr1 rearrangement in which the left junctions of Tlr1 were further to the left than in strain BVII, resulting in the elimination of an additional 187 bp in CU354 or an additional 282 bp in C2-468-3 (Fig. 2A). Thus, although the 1.1-kb HindIII fragment was the most common product of the Tlr-1 rearrangement, there was a discrete set of alternative junction sites.

Long inverted repeats near the TIr1 rearrangement junctions. The most striking feature of the DNA sequence near the rearrangement junctions was a perfect repeat of $751 \mathrm{bp}$ (Fig. 2 ). In view of this characteristic, the rearrangement has been named Tlr1, for Tetrahymena long repeat. Because the DNA between the junctions has not been completely mapped, the relative orientation of the long repeats in the micronuclear genome is unknown. With respect to sequences retained in the macronuclear genome, the repeats are in an inverted orientation. The inverted repeat spans the left junction of the Tlr1 rearrangement and lies within the eliminated DNA at the right junction (see Fig. 5A).

All of the sequences to the BglII end of the fragment which covers the right junction, Tlr1.rB-H, were repeated in the left junction fragment, IIC7 (Fig. 1). In order to determine whether the repeat extended beyond the BglII site, several attempts were made to clone additional sequences at the right junction. These were unsuccessful because of instability of the cloned DNA in a variety of bacterial host strains. Genomic restriction mapping suggested that the inverted repeat did not extend as far as the EcoRI and HindIII sites in the left junction clone, IIC7. Micronuclear DNA between the EcoRI site and the $B g l$ II site inside the right junction of Tlr1 was amplified by PCR, as described in Materials and Methods. Direct sequencing of the PCR product revealed that the inverted repeat extends 74 bp beyond the BglII site, and the total length of the inverted repeat is $825 \mathrm{bp}$ with only three base pairs different between the left and right repeats.

Long inverted repeats are a characteristic feature of elements which undergo DNA-mediated transposition. In general, such elements are repeated in the genome, with copy numbers ranging from 30 to $50(8)$ to $>10^{4}(33)$. In order to obtain an estimate of the copy number of fragments containing sequences with homology to the inverted repeat, Southern blots of genomic DNA were hybridized with the Sau3A-ClaI fragment, IIC7.1b. In macronuclear DNA, only the fragments corresponding in size to Tlr1.1 were observed (data not shown). That is, with the exception of the part of the inverted repeat retained in the macronucleus at the Tlr1 junction, sequences in IIC7.1b are micronucleus specific. Micronuclear DNA was digested with three enzymes. HindIII has no sites within the Tlr1 repeat and was expected to display the full array of fragments with sequences homologous to IIC7.1b. BglII cuts within the inverted repeat (Fig. 2). The hybridizing fragments from Tlr1 contain IIC7.1b and DNA outside the inverted repeat which is retained in the macronucleus (Fig. 2). $R s a$ I also digests within the Tlr1 repeat, to generate fragments in which IIC7.1b is linked to additional micronucleus-specific DNA. Figure 4 shows that DNA digested with these restriction enzymes contains seven or eight fragments with homology to sequences within the inverted repeat. This is in accord with previously published data showing that the IIC7.1b probe hybridized to micronuclear DNA with less intensity than the single-copy IIC7.1a region (48). Thus, the copy number of fragments with homology to IIC7.1b is lower than expected for a family of eukaryotic transposable elements.

Tandem 19-bp repeats within the inverted repeat of TIr1 are associated with DNA rearrangements at other sites in the micronuclear genome. Two different 19-bp tandemly repeated sequences were found within the Tlr1 inverted repeat. The sequence of the first repeat, 19A, is 5'-ATTATTTCTTTTTA CATTT-3'. There are three copies of this repeat followed by a two more degenerate repeats in tandem and a third which is separated from the tandem array by $10 \mathrm{bp}$. The sequence of the second repeated 19-mer, 19B, is 5'-TTTCTCATTTTATG AAAAG-3'. Three perfect copies of 19B are followed by three degenerate repeats (Fig. 2). Both kinds of tandem repeats contain the pentamer TTTCT, which is present 17 times within 


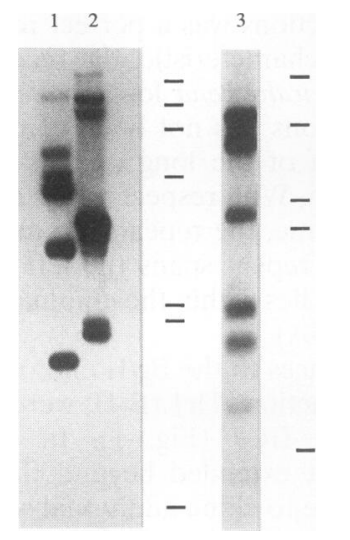

FIG. 4. Southern blot of micronuclear DNA digested with BglII (lane 1), HindIII (lane 2), and RsaI (lane 3) and hybridized with IIC7.1b. Bars represent the mobilities of HindIII restriction fragments of $\lambda$ DNA run as molecular weight standards.

the 398 bp of DNA near the left end of the rearrangement. This is much higher than the expected frequency of one TTTCT in 404 bp. Parts of the inverted repeat, particularly in the region of the 19A repeats, have a strong purine-pyrimidine strand bias.

In-gel hybridization experiments suggested that $19 \mathrm{~A}$ and 19B are associated with each other at several sites in the micronuclear genome. The two 19-mers hybridized to seven fragments of the same mobility in micronuclear DNA digested with either BglII or HindIII (Fig. 5B). 19B hybridized to an additional 3.3-kb HindIII fragment from micronuclear DNA which was not recognized by 19A. One possibility is that there is a HindIII site within the group of tandem 19B repeats at one of the genomic loci. Digestion at that site would produce a fragment which hybridizes to both $19 \mathrm{~A}$ and $19 \mathrm{~B}$ and another fragment with homology to only 19B. A T-to-C transition at the first base of an internal 19B repeat would produce such a HindIII restriction site. The patterns of fragments observed after hybridization of genomic DNA with the 19-mers were very similar to those seen when the DNA was probed with IIC7.1b (Fig. 4).

Not all of the fragments hybridize to 19-mers with equal intensity. At least two possibilities might account for this observation. First, some loci might have more copies of the tandem repeat. Since the hybridizations were done at a stringency chosen to retain only perfect hybrids, this would occur if some of the hybridizing regions contained a repeat similar to those at Tlr1, but lacking the degeneracy. Alternatively, some of the bands in the blot shown in Fig. 5 may represent comigrating fragments. Rehybridization of the oligonucleotide gels with single-copy probes showed comigration of the fragments containing Tlr1.r and Tlr2.1 in DNA digested with BglII (but not HindIII).

The patterns of 19-mer hybridization are different in micronuclear and macronuclear DNA. In macronuclear DNA, 19A hybridized to only one fragment, which was the expected size for the left junction of Tlr1. All of the 19B sequences are eliminated from macronuclear DNA, as they are in the Tlr1 rearrangement. The lack of comigrating fragments in micronuclear DNA and macronuclear DNA indicates that 19A and 19B are associated with DNA rearrangements.

The 19-mer repeats are present within pairs of longer repeated sequences. DNA sequence analysis revealed that $19 \mathrm{~A}$ and 19B are part of the larger (825-bp) Tlr1 inverted repeat

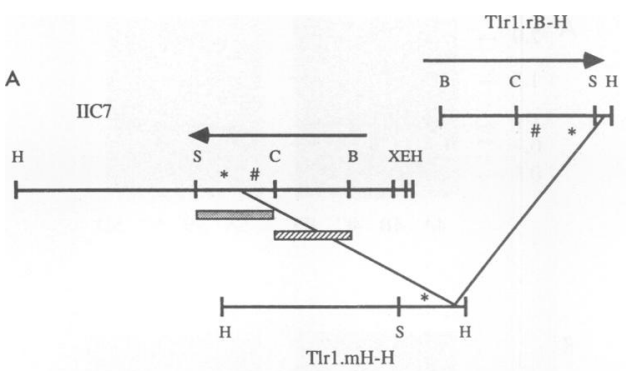

B

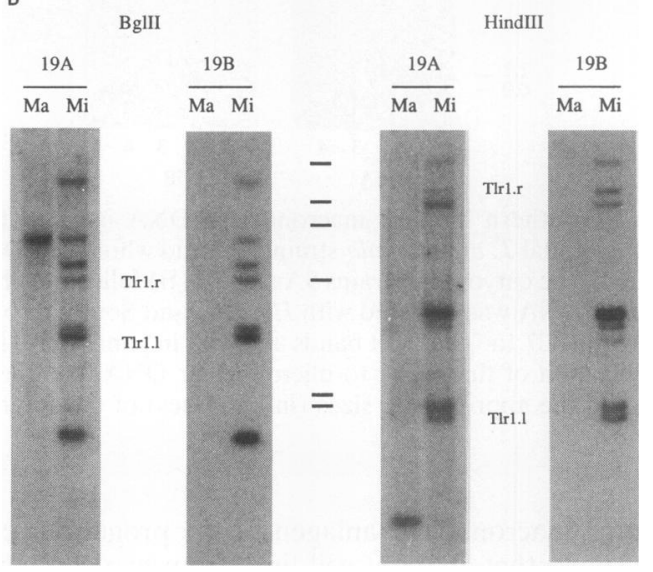

FIG. 5. (A) Maps of the restriction fragments which cover the Tlr1 rearrangement junctions. H, HindIII; S, Sau3A; C, ClaI; B, BglII; X, $X b a \mathrm{I} ; \mathrm{E}, E c o$ RI. Arrows indicate the inverted repeat. Probes used in the experiments shown in Fig. 5B and 6 are as follows: *, 19A tandem repeats; \#, 19B tandem repeats; stippled bar, IIC7.1b; hatched bar, IIC7.2a. (B) Hybridization of end-labelled 19A or 19B to DNA immobilized in gels. Macronuclear (Ma) or micronuclear (Mi) DNA was digested with BglII or HindIII. The indicated bands in micronuclear DNA have been identified as the left and right junctions of Tlr1 by rehybridization of the gels with single-copy probes specific for each family member. The macronuclear fragments are the product of the Tlr1 rearrangement. Bars represent the mobility of HindIII restriction fragments of $\lambda$ DNA run as molecular weight standards.

(Fig. 2). This raised the question of whether the entire Tlr1 inverted repeat is conserved at all of the seven locations in the micronuclear genome that hybridized to the 19-mers. A subclone containing the 19-mers, IIC7.1b, was used to probe micronuclear DNA digested with restriction enzymes that cut within the Tlr1 inverted repeat, Sau3A and ClaI (Fig. 6A). If the long terminal repeat was conserved at other sites in the micronuclear genome, the probe would hybridize to restriction fragments the same size as those from Tlr1. Instead, IIC7.1b hybridization produced multiple bands, suggesting that at least some of the restriction sites within the Tlr1 long inverted repeat are not conserved at every site where there are 19-mer repeats.

Fragments of the expected size for the Tlr1 inverted repeat, a 718-bp Sau3A fragment (Fig. 6A, lane 2), were generated by digestion with Sau3A at the Sau3A and BglII sites within the inverted repeat. That fragment was further digested by $\mathrm{ClaI}$ to generate the 394-bp fragment Sau3A-ClaI fragment (Fig. 6A, lane 1), confirming the presence of the ClaI site in Tlr1.

Hybridization of Sau3A-digested DNA with IIC7.1b resulted in two major bands in addition to the one derived from the Tlr1 inverted repeats. Since there are at least two 718-bp 


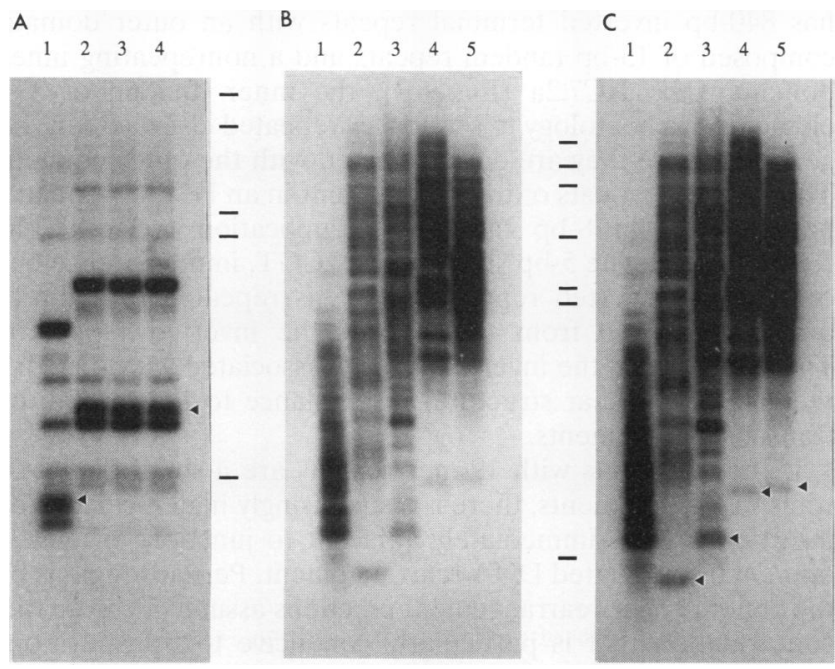

FIG. 6. Southern blots of micronuclear DNA digested with restriction enzymes and probed with subclones of the Tlr1 inverted repeat. (A) Probe was IIC7.1b (Fig. 5A). Restriction enzymes were as follows: lane 1, ClaI and Sau3A; lane 2, Sau3A; lane 3, Sau3A and XbaI; lane 4, Sau3A and EcoRI. (B and C) Probe was IIC7.2a (Fig. 5A). Restriction enzymes were as follows: lane 1, Sau3A; lane 2, ClaI and $B g l \mathrm{II}$; lane 3, ClaI and $X b a \mathrm{I}$; lane 4,ClaI and EcoRI; lane 5, ClaI and HindIII. Exposure was for $38 \mathrm{~h}$ (A), $20 \mathrm{~h}$ (B), and $48 \mathrm{~h}$ (C). The fragments indicated by arrows are the sizes expected for Tir1. Bars represent the mobility of HindIII restriction fragments of $\lambda$ DNA run as molecular weight standards. Panel A shows only the five smallest fragments.

Sau 3 A restriction fragments from the Tlr1 inverted repeat and because the hybridization intensities of the three major bands in lane 2 of Fig. 5 were similar, we propose that each of the three prominent bands in Fig. $6 \mathrm{~A}$, lanes 2,3 , and 4, may contain a pair of comigrating restriction fragments. This hypothesis was supported by the observation that the $\sim 650$-bp Sau3A fragment (Fig. 6A, lane 2) was reduced in intensity by half upon digestion with ClaI (Fig. 5A, lane 1). This suggested that the $\sim 650$-bp band generated by Sau3A resulted from two restriction fragments, only one of which contained a ClaI site. The data support a model in which the 19-mers are located within pairs of longer repeated sequences.

Although the inverted repeat containing the 19-mers is not perfectly conserved, it may be conserved to a large extent with some restriction site polymorphism. It is notable that the largest band in the Sau3A digest probed with IIC7.1b, which is about $1.4 \mathrm{~kb}$, is reduced in size by about $300 \mathrm{bp}$ upon Cla I digestion (Fig. 6A, compare lanes 1 and 2), suggesting that the region between the internal BglII and ClaI sites is of the Tlr1 inverted repeat is conserved and linked to 19-mer tandem repeats. Assuming that the $B g l I I$ site is conserved, polymorphisms in the Sau3A site could account for the three major Sau3A fragments (Fig. 6A, lane 2). In fact, a clone of micronuclear DNA containing 19-mer repeats other than those from Tlr1 has been isolated. That clone, designated Tlr2.1, has sequence identical to Tlr1 from the ClaI to the RsaI sites of IIC7.1b and diverges before the Sau3A site (Fig. 2A).

In order to investigate the conservation of sequences between the ClaI and BglII sites of the inverted repeat, the IIC7.2a fragment was used to probe Southern blots of micronuclear DNA (Fig. 6B). Discrete bands of the sizes expected from IIC7, the 325-bp ClaI-BglII (lane 2), the 589-bp ClaI$X b a I$ (lane 3), the 675-bp ClaI-EcoRI (lane 4), and the 708-bp

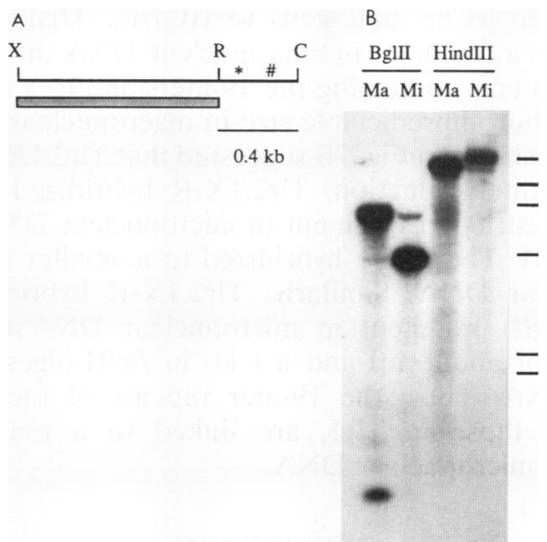

FIG. 7. (A) Restriction map of Tlr2.1X-C. X, XbaI; R, RsaI; C, ClaI. The shaded bar is the Tr2.1X-R subclone used to probe the blot shown in panel B. *, 19A repeats; \#, 19B repeats. (B) Southern blots of macronuclear (Ma) and micronuclear (Mi) DNA digested with BglII or HindIII and probed with Tlr2.1X-R. Minor bands in the micronuclear DNA lanes which comigrate with the fragments in macronuclear DNA are due to the contamination of the micronuclear DNA preparations with macronuclear DNA. Bars to the right of the Southern blot represent the mobilities of HindIII restriction fragments of $\lambda$ DNA run as molecular weight standards.

ClaI-HindIII (lane 5), are indicated by the arrows in Fig. 6C. However, the probe also hybridized to many additional restriction fragments. In all, approximately 30 bands were resolved, showing that sequences with homology to IIC7.2a are present at a higher copy number than the sequences with homology to the outer region of the Tlr1 inverted repeat containing the 19-mers.

19-mer repeats are proximal to a second rearrangement junction, TIr2. The experiment whose results are given in Fig. 5 showed that $19 \mathrm{~A}$ and 19B were linked to each other and associated with rearrangements at seven sites in the micronuclear genome. The data in Fig. 6 suggested that the 19-mers were located within pairs of longer repeated sequences. Our working hypothesis is that the Tlr1 rearrangement is a member of a small family of DNA rearrangements with long terminal repeats containing the 19 -bp tandem repeats near the rearrangement junctions.

If the micronucleus-specific 19-mers are near rearrangement junctions they should be in close proximity to DNA sequences which are retained in the macronuclear genome. A clone designated Tlr2.1X-C (Fig. 7A) was selected from a plasmid library of micronuclear DNA fragments on the basis of hybridization to the subclone IIC7.1b, which contains the 19-mer repeats (Fig. 5A). The presence of the 19-mers on the clone was verified by sequence analysis. The sequence of Tlr2.1X-C was identical to the region of the Tlr1 inverted repeat from the ClaI site 1217 (Fig. 2A) to the Rsa I site 830 and diverged at bp 829. The sequence was confirmed by sequencing the opposite strand from the Tlr2.1R-C subclone and by sequencing of the Tlr2.1X-C clone across the $R s a I$ site from an internal primer. The sequencing data for Tlr 2 were consistent with the data in Fig. 6A, which suggested that the Sau3A site adjacent to the RsaI site in Tlr1 was not conserved among the long repeats containing the 19-mers.

In order to obtain a probe of unique DNA from Tlr2.1X-C, the 19-mer repeats were removed by subcloning the XbaI-RsaI fragment Tlr2.1X-R (Fig. 7A). If the 19-mers in Tlr2.1X-C were proximal to a DNA rearrangement junction, Tlr2.1X-R 
was expected to be analogous to IIC7.1a. That is, it would hybridize to a fragment in micronuclear DNA the size of one of the fragments containing the 19-mers and to a fragment of a different but unpredictable size in macronuclear DNA.

The data shown in Fig. 7B suggested that Tlr2.1X-C contains a rearrangement junction. Tlr2.1X-R hybridized to a large $(>23-\mathrm{kb})$ restriction fragment in micronuclear DNA digested with HindIII. The probe hybridized to a smaller fragment in macronuclear DNA. Similarly, Tlr2.1X-R hybridized to an 4.4-kb BglII fragment in micronuclear DNA and to two fragments of about 1.0 and $6.4 \mathrm{~kb}$ in $B g l \mathrm{II}$-digested macronuclear DNA. Thus, the $19-$ mer repeats of the Tlr2.1X-C clone, like those in Tlr1, are linked to a rearrangement junction in micronuclear DNA.

\section{DISCUSSION}

TIr1 is a novel DNA rearrangement in $T$. thermophila. The distance between the rearrangement junctions of Tlr1 is greater than for developmentally regulated DNA rearrangements previously described for $T$. thermophila. The four deletion-ligation rearrangements analyzed to date result in the elimination of $600 \mathrm{bp}$ to $2.9 \mathrm{~kb}$ of DNA from the macronucleus $(2,4,5,26,37)$. The Tlr1 rearrangement joins sequences which are separated by $13 \mathrm{~kb}$ or more in the micronuclear genome. Since the Tlr1 rearrangement is intrachromosomal and the DNA adjacent to both rearrangement junctions is micronucleus specific, the simplest interpretation of the data is that Tlr1 deletes a single, contiguous stretch of DNA. Clones containing 10 to $15 \mathrm{~kb}$ of micronucleus-limited DNA have been isolated in several laboratories $(12,29,61)$, suggesting that long stretches of micronucleus-limited DNA are not uncommon. However, since the fate of most of the sequences between the Tlr1 junction sites is unknown, we cannot exclude the possibility of a more complex series of events.

A second difference between Tlr1 and rearrangements previously characterized for $T$. thermophila is the close proximity of the rearrangement junctions to long inverted repeats. The 19-mers within the repeat constitute a small family of micronucleus-specific sequences (Fig. 5). The exact size of the family is unknown, but it is likely to contain about six to eight members altogether (Fig. 4), which are located within longer repeats (Fig. 6). A perfect copy of 393 bp of DNA from the region of the Tlr1 inverted repeat containing the 19-mers was found very close to the junction of a second rearrangement, designated Tlr2 (Fig. 7).

Transposable element or cis-acting signal for developmentally regulated DNA rearrangement? cis-acting signals have been identified for only one of the five developmentally regulated rearrangements studied to date in $T$. thermophila. Ten-base-pair $A_{5} G_{5}$ polypurine tracts near the junctions of the $M$ rearrangement have been shown to be both necessary and sufficient $c i s$-acting sequences $(5,23,24)$. Since the other four rearrangements in $T$. thermophila analyzed to date all lack $\mathrm{A}_{5} \mathrm{G}_{5}$ sequences, the polypurine tracts cannot be the universal signal for DNA rearrangement in $T$. thermophila. The most striking structural feature in the vicinity of the Tlr1 rearrangement junctions is the 825 -bp inverted repeat. The association of the inverted repeat with at least two different rearrangement junctions suggests that the repeat contains cis-acting signals for DNA rearrangement.

An alternative interpretation of the data is that Tlr1 is a member of a very small family of transposable elements. Although there is to date no evidence for transposition, the long inverted repeat of Tlr1 bears a striking resemblance to the TU elements in sea urchins. The canonical TU element, TU1, has 840-bp inverted terminal repeats with an outer domain composed of 15-bp tandem repeats and a nonrepeating inner domain. Like IIC7.2a (Fig. 6B), the inner domain of TU elements has homology to sequences repeated elsewhere in the genome where they are not associated with the outer domain. The inverted repeats of the TU element in an H2B orphon are bounded by an 8-bp target site duplication (reviewed in reference 28). The 5-bp sequence, CTCGT, immediately adjacent to the inverted repeat in IIC7, is repeated in a direct orientation $1 \mathrm{bp}$ from the end of the inverted repeat in Tlr1.rB-H. Thus, the inverted repeats associated with the Tlr1 rearrangement bear structural resemblance to the termini of transposable elements.

If the fragments with 19-mer repeats are a small family of transposable elements, there is a surprisingly high incidence of insertion at sites immediately adjacent to junctions of developmentally regulated DNA rearrangement. Perhaps regions of the genome near rearrangement junctions assume a chromatin conformation that is particularly conducive to transposition. The preferential (though not exclusive) insertion of yeast Ty elements near the $5^{\prime}$ ends of active genes suggests that open chromatin is particularly receptive to insertion of Ty elements (9).

Relationship between developmentally regulated DNA rearrangements and transposons. A relationship between developmentally regulated DNA rearrangement and transposition of various elements has been suggested by the structure of the elements themselves and of their excision products. The first of these were the telomere-bearing elements (TBE) of Oxytricha fallax and the transposon-like elements (Tel) of T. thermophila $(19,27)$. These elements are repeated in the respective micronuclear genomes and have terminal inverted repeats $(77$ or 78 bp for TBE and $30 \mathrm{bp}$ for Tel) which end in sequences corresponding to those of the telomeres of the respective organism. This structure led to the suggestion that the elements were inserted into the micronuclear genome as a result of transposition events. It was proposed that they existed at some stage as extrachromosomal linears which acquired telomeres before they were integrated in the micronuclear genome. Since the bulk of the excised TBEs are excised as circular molecules, it was further proposed that transposase activity is modified in the developing macronuclear anlagen in order to function in DNA excision (52).

The vast majority of the gene-size macronuclear DNA molecules in the hypotrichs analyzed to date are formed by the removal of internal eliminated sequences (IES), so named because they are internal to macronucleus-destined sequences in the micronuclear genome and are eliminated during macronuclear development (38). These are generally short, 14- to 500-bp sequences which are single copy in the micronucleus (46). In at least two cases, TBEs are IESs; they are precisely excised from a macronucleus-destined sequence $(30,52)$. This has led Herrick and his colleagues to propose the unifying hypothesis that all IESs in the hypotrichs are transposon derived and the short IESs are transposon remnants $(27,30)$. In this view, the ciliates have evolved a mechanism that recognizes and precisely excises transposons from the transcriptionally active macronucleus.

There is precedent for association of long inverted repeats with genome rearrangement in ciliates. Tec-1 and Tec- 2 (for transposon-like element, Euplotes crassus) are highly repeated elements in the micronuclear genome of the hypotrich $E$. crassus. These 5.3-kb elements have 700 - to 800 -bp terminal inverted repeats and are preferentially located near macronucleus-destined sequences $(6,33,41)$. Although Tec elements and TBEs have open reading frames that bear sequence 
similarity to the Tc1-IS630 family of transposases $(21,32)$, transcripts of the Tec open reading frame are not detected in amounts sufficient to account for the massive excision of these elements (34). Striking similarities in the structure of the excision products of Tec elements and the IESs of $E$. crassus have led to the suggestion that they share at least some components of the excision machinery $(33,35,39,49)$.

The presence of the long repeats near both the Tlr1 and Tlr2 rearrangement junctions suggests that they contain cis-acting sequences for DNA rearrangement. The similarity between the structure of these sequences and that of transposons leads us to further propose that such DNA rearrangements may have been the precursors of some of the transposable elements. In the ciliates, elements such as the TBEs and the Tec elements may arise from sequences involved in DNA rearrangement. These sequences may become associated with transposase-like functions, which are either endogenous or imported by horizontal transmission. Eventually such sequences may evolve into independent, highly repeated, transposable elements. This model predicts that in some systems there will be elements which structurally resemble transposons but have not acquired the ability to transpose. That is, although they undergo deletion during development of the macronucleus, no mechanism has evolved for insertion at a new site. The DNA deleted at Tlr1 may be an example of such an element.

Transposable elements have a negative selective value as mutagens. In order for them to evolve, this negative selective pressure must be counterbalanced by positive selection conferred either by the transposon itself or by the cellular process which produced and/or maintains them. The selective advantage of DNA rearrangement in the ciliates is not known, but it has been suggested that micronucleus-specific sequences act as negative regulatory sequences in the micronuclear genome of $T$. thermophila $(15,37,57)$ or that variable DNA rearrangement might provide for genetic diversity $(29,43,44)$ as it does for the mammalian immunoglobulin genes (50) and the trypanosome surface antigens (22).

There is good evidence that transposition plays a role in maintenance of chromosome structure. The heterochromatic Het-A element (50a) is located at the telomeres of Drosophila chromosomes and is added to broken chromosome ends (7, 51). Het-A bears structural resemblance to the class II retrotransposons, such as the mammalian LINE elements (31). Because no evidence has been found that Het-A encodes its own transposase, Pardue and her colleagues have suggested that the transposition function is normally supplied by a gene acting in trans and that elements which capture such a gene then escape restrictions on chromosomal location and mobility (45).

Transposable elements may take advantage of molecular machinery which was originally dedicated to cellular events other than DNA rearrangement and transposition. In Drosophila melanogaster, there are two types of pole cells. Only those which migrate to the germ line and differentiate into germ cells splice the third $P$ element intron (40). Kobayashi et al. suggested that an RNA splicing activity required for germ line development also is used in $\mathrm{P}$ element transposition. Several types of transposons, such as the Ty elements in Saccharomyces cerevisiae, transpose through an RNA intermediate (10). These elements presumably require reverse transcriptase as part of the transposition machinery, which might have evolved from reverse transcriptases needed for cellular processes such as telomere synthesis (42). Thus, transposons may survive in the eukaryotic genome despite a negative selective value because they take advantage of cellular machinery which is required for a variety of other, indispensable, cellular processes. Occasional generation of new transposons may contribute to the observed inconsistencies between the phylogenies of transposons and the phylogenies of their host species (17).

\section{ACKNOWLEDGMENTS}

We are grateful to Inta Kalve for technical assistance and to Elizabeth Capowski for helpful discussion.

The project was supported by Public Health Service grant GM39890 from the National Institutes of Health and by grant MCB-9110651 from the National Science Foundation. K.M.K. is a Clare Boothe Luce Professor and gratefully acknowledges the support of the Henry Luce Foundation. J.M.W. was supported in part by an NIH predoctoral training grant.

\section{REFERENCES}

1. Allitto, B. A., and K. M. Karrer. 1986 . A family of DNA sequences is reproducibly rearranged in the somatic nucleus of Tetrahymena. Nucleic Acids Res. 14:8007-8025.

2. Austerberry, C. F., C. D. Allis, and M.-C. Yao. 1984. Specific DNA rearrangements in synchronously developing nuclei of Tetrahymena. Proc. Natl. Acad. Sci. USA 81:7383-7387.

3. Austerberry, C. F., R. O. Snyder, and M.-C. Yao. 1989. Sequence microheterogeneity is generated at junctions of programmed DNA deletions in Tetrahymena thermophila. Nucleic Acids Res. 17:7263-7272.

4. Austerberry, C. F., and M.-C. Yao. 1987. Nucleotide sequence structure and consistency of a developmentally regulated DNA deletion in Tetrahymena thermophila. Mol. Cell. Biol. 7:435443.

5. Austerberry, C. F., and M.-C. Yao. 1988. Sequence structures of two developmentally regulated, alternative DNA deletion junctions in Tetrahymena thermophila. Mol. Cell. Biol. 8:3947-3950.

6. Baird, S. E., F. M. Fino, S. L. Tausta, and L. A. Klobutcher. 1989. Micronuclear genome organization in Euplotes crassus: a transponsonlike element is removed during macronuclear development. Mol. Cell. Biol. 9:3793-3807.

7. Biessmann, H., J. M. Mason, K. Ferry, M. d'Hulst, K. Valgeirsdottir, K. L. Traverse, and M. L. Pardue. 1990. Addition of telomere-associated HeT DNA sequences "heals" broken chromosome ends in Drosophila. Cell 61:663-673.

8. Bingham, P. M., M. G. Kidwell, and G. M. Rubin. 1982. The molecular basis of P-M hybrid dysgenesis: the role of the $P$ element, a P-strain-specific transposon family. Cell 29:9951004.

9. Boeke, J. D. 1989. Transposable elements in Saccharomyces cerevisiae, p. 335-374. In D. E. Berg and M. M. Howe (ed.), Mobile DNA. American Society for Microbiology, Washington, D.C.

10. Boeke, J. D., D. J. Garfinkel, C. A. Styles, and G. R. Fink. 1985. Ty elements transpose through an RNA intermediate. Cell 40:491500.

11. Brunk, C. F., and R. K. Conover. 1985. Elimination of micronuclear specific DNA sequences early in anlagen development. Mol. Cell. Biol. 5:93-98.

12. Brunk, C. F., G. S. Tsao, C. H. Diamond, P. S. Ohashi, N. G. N. Tsao, and R. E. Pearlman. 1982. Reorganization of unique and repetitive sequences during nuclear development in Tetrahymena thermophila. Can. J. Biochem. 60:847-853.

13. Bruns, P. J. 1986. Genetic organization of Tetrahymena, p. 27-44. In J. G. Gall (ed.), The molecular biology of ciliated protozoa. Academic Press, Inc., Orlando, Fla.

14. Bruns, P. J., T. B. Brussard, and E. V. Merriam. 1983. Nullisomic Tetrahymena. II. A set of nullisomics define the germinal chromosomes. Genetics 104:257-270.

15. Callahan, R. C., G. Shalke, and M. A. Gorovsky. 1984. Developmental rearrangements associated with a single type of expressed $\alpha$-tubulin gene in Tetrahymena. Cell 36:441-445.

16. Capowski, E. E., J. M. Wells, G. S. Harrison, and K. M. Karrer. 1989. Molecular analysis of methylation patterns in Tetrahymena thermophila. Mol. Cell. Biol. 9:2598-2605.

17. Capy, P., D. Anxolabehere, and T. Langin. 1994. The strange 
phylogenies of transposable elements: are horizontal transfers the only explanation? Trends Genet. 10:7-12.

18. Cassidy-Hanley, D., M.-C. Yao, and P. J. Bruns. 1994. A method for mapping germ line sequences in Tetrahymena thermophila using the polymerase chain reaction. Genetics 137:95-106.

19. Cherry, J. M., and E. H. Blackburn. 1985. The internally located telomeric sequences in the germ-line chromosomes of Tetrahy mena are at the ends of transposon-like elements. Cell 43:747758

20. DiLella, A. G., and S. Woo. 1987. Hybridization of genomic DNA to oligonucleotide probes in the presence of tetramethylammonium chloride. Methods Enzymol. 152:447-451.

21. Doak, T. G., F. P. Doerder, C. L. Jahn, and G. Herrick. 1994. A proposed superfamily of transposase-related genes: new members in transposon-like elements of ciliated protozoa and a common "D35E" motif. Proc. Natl. Acad. Sci. USA 91:942-946.

22. Donelson, J. E. 1989. DNA rearrangements and antigenic variation in African tryopanosomes, p. 763-781. In D. E. Berg and M. M. Howe (ed.), Mobile DNA. American Society for Microbiology, Washington, D.C.

23. Godiska, R., and M.-C. Yao. 1990. A programmed site-specific DNA rearrangement in Tetrahymena thermophila requires flanking polypurine tracts. Cell 61:1237-1246.

24. Godiska, R., and M.-C. Yao. 1993. A distant 10-bp sequence specifies the boundaries of a programmed DNA deletion in Tetrahymena. Genes Dev. 7:2357-2365.

25. Gorovsky, M. A., M.-C. Yao, J. B. Keevert, and G. L. Pleger. 1975. Isolation of micro- and macronuclei of Tetrahymena pyriformis Methods Cell Biol. 9:311-327.

26. Heinonen, T. Y. K., and R. E. Pearlman. 1994. A germ line specific element in an intron in Tetrahymena thermophila. J. Biol. Chem. 269:17428-17433

27. Herrick, G. S. Cartinhour, D. Dawson, D. Ang, R. Sheets, A. Lee, and $K$. Williams. 1985. Mobile elements bounded by C4A4 telomeric repeats in Oxytricha fallax. Cell 43:759-768.

28. Hoffman-Liebermann, B., D. Liebermann, and S. N. Cohen. 1989 TU elements and puppy sequences, p. 575-592. In D. E. Berg and M. M. Howe (ed.), Mobile DNA. American Society for Microbiology, Washington, D.C.

29. Howard, E. A., and E. H. Blackburn. 1985. Reproducible and variable genomic rearrangements occur in the developing somatic nucleus of the ciliate Tetrahymena thermophila. Mol. Cell. Biol. 5:2039-2050.

30. Hunter, D. J., K. Williams, S. Cartinhour, and G. Herrick. 1989. Precise excision of telomere-bearing transposons during Oxytricha fallax macronuclear development. Genes Dev. 3:2101-2112.

31. Hutchison, C. A., III., S. C. Hardies, D. D. Loeb, W. R. Shehee, and M. H. Edgell. 1989. LINES and related retroposons: long interspersed repeated sequences in the eucaryotic genome, p. 593617. In D. E. Berg and M. M. Howe (ed.), Mobile DNA. American Society for Microbiology, Washington, D.C.

32. Jahn, C. L., S. Z. Doktor, J. S. Frels, J. W. Jaraczewski, and M. F. Krikau. 1993. Structures of the Euplotes crassus Tec1 and Tec2 elements: identification of putative transposase coding regions. Gene 133:71-78.

33. Jahn, C. L., M. F. Krikau, and S. Shyman. 1989. Developmentally coordinated en masse excision of a highly repetitive element in E. crassus. Cell 59:1009-1018.

34. Jaraczewski, J. W., J. S. Frels, and C. L. Jahn. A developmentally regulated, low abundance Tec element transcript in Euplotes crassus-implications for DNA elimination and transposition. Submitted for publication.

35. Jaraczewski, J. W., and C. L. Jahn. 1993. Elimination of Tec elements involves a novel excision process. Genes Dev. 7:95-105.

36. Karrer, K. M. 1983 . Germ line-specific DNA sequences are present on all five micronuclear chromosomes in Tetrahymena thermophila. Mol. Cell. Biol. 3:1909-1919.

37. Katoh, M., M. Hirono, T. Takemasa, M. Kimura, and Y. Watanabe. 1993. A micronucleus-specific sequence exists in the 5 -upstream region of calmodulin gene in Tetrahymena thermophila. Nucleic Acids Res. 21:2409-2414.

38. Klobutcher, L. A., C. L. Jahn, and D. M. Prescott. 1984. Internal sequences are eliminated from genes during macronuclear devel- opment in the ciliated protozoan Oxytricha nova. Cell 36:10451055.

39. Klobutcher, L. A., L. R. Turner, and J. LaPlante. 1993. Circular forms of developmentally excised DNA in Euplotes crassus have a heteroduplex junction. Genes Dev. 7:84-94.

40. Kobayashi, S., T. Kitamura, H. Sasaki, and M. Okada. 1993. Two types of pole cells are present in the Drosophila embryo, one with and one without splicing activity for the third P-element intron. Development 117:885-893

41. Krikau, M. F., and C. L. Jahn. 1991. Tec2, a second transposonlike element demonstrating developmentally programmed excision in Euplotes crassus. Mol. Cell. Biol. 11:4751-4759.

42. Lundblat, V., and E. H. Blackburn. 1990. RNA-dependent polymerase motifs in EST1: tentative identification of a protein component of an essential yeast telomerase. Cell 60:529-530.

43. Orias, E. 1981. Probable somatic DNA rearrangements in mating type determination in Tetrahymena thermophila: a review and a model. Dev. Genet. 2:185-202.

44. Orias, E., and M. P. Baum. 1985. Mating type differentiation in Tetrahymena thermophila: characterization of the delayed refeeding effect and its implications concerning intranuclear coordination. Dev. Genet. 5:141-156.

45. Pardue, M.-L. 1992. Do some "parasitic" DNA elements earn and honest living?, p. 145-154. In N. Federoff and D. Botstein (ed.), The dynamic genome: Barbara McClintock's ideas in the century of genetics. Cold Spring Harbor Laboratory Press, Cold Spring Harbor, N.Y.

46. Ribas-Aparicio, R. M., J. J. Sparkowski, A. E. Proulx, J. D. Mitchell, and L. A. Klobutcher. 1987. Nucleic acid splicing events occur frequently during macronuclear development in the protozoan Oxytricha nova and involve the elimination of unique DNA. Genes Dev. 1:323-336.

47. Sambrook, J. E. F. Fritsch, and T. Maniatis. 1989. Molecular cloning: a laboratory manual, 2nd ed. Cold Spring Harbor Laboratory Press, Cold Spring Harbor, N.Y.

48. Stein-Gavens, S., J. M. Wells, and K. M. Karrer. 1987. A germ line specific DNA sequence is transcribed in Tetrahymena. Dev. Biol. 120:259-269.

49. Tausta, S. L., and L. A. Klobutcher. 1989. Detection of circular forms of eliminated DNA during macronuclear development in $\mathrm{E}$. crassus. Cell 59:1019-1026.

50. Tonegawa, S. 1983. Somatic generation of antibody diversity. Nature (London) 302:575-581.

50a.Traverse, K. L., and M. L. Pardue. 1990. HeT DNA: a family of mosaic repeated sequences specific for heterochromatin in Drosophila. Proc. Natl. Acad. Sci. USA 87:7998-8002.

51. Traverse, K. L., and M. L. Pardue. 1988. A spontaneously opened ring chromosome of Drosophila has acquired He-T DNA sequences on both new telomeres. Proc. Natl. Acad. Sci. USA 85:8116-8120.

52. Williams, K., T. G. Doak, and G. Herrick. 1993. Developmental precise excision of Oxytricha trifallax telomere-bearing elements and formation of circles closed by a copy of the flanking target duplication. EMBO J. 12:4593-4602.

53. Wood, W. I., J. Gitschier, L. A. Lasky, and R. M. Lawn. 1985. Base composition-independent hybridization in tetramethylammonium chloride: a method for oligonucleotide screening of highly complex gene libraries. Proc. Natl. Acad. Sci. USA 82:1585-1588.

54. Wyman, A. R., and K. F. Wertman. 1987. Host strains that alleviate underrepresentation of specific sequences: overview. Methods Enzymol. 152:173-180.

55. Yao, M.-C. 1981. Ribosomal RNA gene amplification in Tetrahymena may be associated with chromosome breakage and DNA elimination. Cell 24:765-774.

56. Yao, M.-C. 1989. Site-specific chromosome breakage and DNA deletion in ciliates, p. 715-731. In D. E. Berg and M. M. Howe (ed.), Mobile DNA. American Society for Microbiology, Washington, D.C.

57. Yao, M.-C., J. Choi, S. Yokoyama, C. F. Austerberry, and C.-H. Yao. 1984. DNA elimination in Tetrahymena: a developmental process involving extensive breakage and rejoining of DNA at defined sites. Cell 36:433-440.

58. Yao, M.-C., and M. Gorovsky. 1974. Comparison of the sequences 
of macronuclear and micronuclear DNA of Tetrahymena pyriformis. Chromosoma 48:1-18.

59. Yao, M.-C., C.-H. Yao, and B. Monks. 1990. The controlling sequence for site-specific chromosome breakage in Tetrahymena. Cell 63:763-772.

60. Yao, M.-C., K. Zheng, and C.-H. Yao. 1987. A conserved nucle- otide sequence at the sites of developmentally regulated chromosome breakage in Tetrahymena. Cell 48:779-788.

61. Yokoyama, R., and M.-C. Yao. 1984. Internal micronuclear DNA regions which include sequences homologous to macronuclear telomeres are deleted during development in Tetrahymena. Nucleic Acids Res. 12:6103-6116. 